\title{
Prevalência da Síndrome de Burnout em uma Amostra de Professores Universitários Brasileiros
}

\section{Prevalence of Burnout Syndrome in a Sample of Brazilian University Professors}

\author{
Ludmila da Silva Tavares Costa*, ${ }^{a}$, Pedro Rafael Gil-Monte ${ }^{b}$, Rosana de Fátima Possobon ${ }^{a}$ \\ \& Glaucia Maria Bovi Ambrosano ${ }^{a}$ \\ ${ }^{a}$ Universidade Estadual de Campinas, Piracicaba, São Paulo, Brasil \\ $\&^{b}$ Universitat de València, València, Espanha
}

\begin{abstract}
Resumo
O docente integra um grupo de risco para o desenvolvimento da Síndrome de Burnout (SB). Este estudo investigou a prevalência da SB em 169 professores universitários da cidade de Piracicaba-SP, por meio do Questionário de Avaliação para a Síndrome de Burnout (CESQT versão brasileira). O valor de consistência interna alfa de Cronbach foi satisfatório para todas as dimensões do questionário. Os resultados mostraram que 11,2\% dos professores apresentaram Perfil 1 e $3 \%$ Perfil 2 da SB. A prevalência encontrada é motivo de preocupação e merece atenção, não só pelos danos que a SB provoca na saúde física, mental e social do profissional, mas também pela influência na qualidade de ensino praticado nas escolas.

Palavras-chave: Burnout, saúde ocupacional, professores, prevalência.

Abstract

Professors are part of a risk group for the development of Burnout Syndrome. This study investigated the prevalence of burnout in 169 university professors in Piracicaba-SP through the Burnout Inventory (Brazilian version). The value of Cronbach's alpha was satisfactory for all dimensions of the questionnaire. The results showed that $11.2 \%$ of the professors showed Profile 1 and $3 \%$ Profile 2 of burnout. This prevalence of burnout is cause for concern and deserves attention, not only for the damage it causes at physical, mental and social training, but also for the influence on the quality of education offered.

Keywords: Burnout, occupational health, professors, prevalence.
\end{abstract}

A Síndrome de Burnout (SB) é um elemento de grande relevância dentro do contexto da prevenção de riscos laborais e da análise das condições de trabalho, visto que se encontra vinculada a grandes custos organizacionais e pessoais. Os autores Batista, Carlotto, Coutinho e Augusto (2010) destacam que SB é uma questão de saúde pública devido às suas implicações para a saúde física, mental e social dos indivíduos. Atualmente, a SB é considerada um dos agravos ocupacionais de caráter psicossocial mais importante na sociedade (Melamed, Shirom, Toker, Berliner, \& Shapira, 2006).

\footnotetext{
* Endereço para correspondência: Departamento de Odontologia Social, Universidade Estadual de Campinas, Faculdade de Odontologia de Piracicaba, Avenida Limeira, 901, Areião, Piracicaba, SP, Brasil, 13414-903. E-mail: ludtavares@yahoo.com.br, Pedro.Gil-Monte@uv.es, possobon@fop.unicamp.bre glaucia@fop.unicamp.br. Agradecimento: Os autores agradecem à Coordenação de Aperfeiçoamento de Pessoal de Nível Superior (CAPES) por ter subvencionado o projeto que originou o presente estudo, com bolsa do Programa de Doutorado Sanduíche no Exterior (PDSE - Processo n 9867-11-2) concedida à primeira autora.
}

No Brasil, o Decreto n ${ }^{\circ} 3.048$, de 6 de maio de 1999, aprovou o Regulamento da Previdência Social e, em seu Anexo II, trata dos Agentes Patogênicos causadores de Doenças Profissionais. O item XII da tabela de Transtornos Mentais e do Comportamento Relacionados com o Trabalho (Grupo V da Classificação Internacional das Doenças - CID-10) cita a "Sensação de Estar Acabado" ("Síndrome de Burnout", "Síndrome do Esgotamento Profissional") como sinônimos do Burnout, que, na CID-10, recebe o código Z73.0 (Ministério da Saúde, 2001).

Gil-Monte (2005a) define SB como uma resposta ao estresse laboral crônico, característica dos profissionais que trabalham com pessoas. Os indivíduos apresentam deterioração cognitiva (perda de motivação e baixa realização pessoal no trabalho - baixa Ilusão pelo trabalho) e afetiva (esgotamento emocional e físico - Desgaste psíquico) e, consequentemente, passam a desenvolver atitudes e condutas negativas frente aos clientes e à organização (comportamentos de indiferença, frieza e distanciamento - Indolência). O surgimento de sentimentos de culpa é posterior a esses sintomas, mas não ocorre necessariamente em todos os indivíduos. Desta maneira, é possível distinguir dois 
perfis no processo de SB. O Perfil 1 refere-se ao surgimento de um conjunto de sentimentos e condutas vinculadas ao estresse laboral, que dá origem a uma forma moderada de mal-estar, mas que não incapacita o indivíduo para o exercício do seu trabalho, ainda que pudesse realizá-lo de melhor forma. Este perfil caracteriza-se pela presença de baixos níveis de Ilusão pelo trabalho com altos níveis de Desgaste psíquico e Indolência. O Perfil 2 define os casos clínicos mais deteriorados pelo desenvolvimento da SB, incluindo, além dos sintomas já mencionados, sentimentos de Culpa (Gil-Monte, 2005a).

Como em outros ambientes de trabalho, a SB na educação é um fenômeno complexo e multidimensional, resultante da interação entre aspectos individuais e o ambiente de trabalho (Carlotto, 2002). Esta condição tem repercussões importantes no sistema educacional e na qualidade da aprendizagem (Batista et al., 2010). Para a Organização Internacional do Trabalho (OIT, 1981) a profissão docente é considerada como uma das mais estressantes, com forte incidência de elementos que conduzem à SB. A SB em profissionais de ensino foi avaliada em diversos países como Brasil (Gil-Monte, Carlotto, \& Câmara, 2011), China (Luk, Chan, Cheong, \& Ko, 2010), Portugal (Figueiredo-Ferraz, Gil-Monte, \& Grau-Alberola, 2009), México (Gil-Monte, Unda, \& Sandoval, 2009), França (Vercambre, Brosselin, Gilbert, Nerrière, \& Kovess-Masféty, 2009) e, os autores sugerem novas avaliações e intervenções nesta população de risco.

A sobrecarga laboral é, de acordo com Antoniou, Polychroni e Vlachakis (2006), a principal condição do trabalho docente que desencadeia a SB. Outros autores destacam, como fatores desencadeantes da SB, o conflito de papéis (Burke, Greenglass, \& Schwarzer, 1996), a ambiguidade de papéis (Kokkinos, 2007), conflitos, relacionamentos e comportamento dos alunos (Betoret, 2006; Carlotto, 2002; Hakanen, Bakker, \& Schaufeli, 2005), conflitos com outros professores (Skaalvik \& Skaalvik, 2007) ou falta de apoio social (Marqués-Pinto, Lima, \& Lopes da Silva, 2005).

A prevalência da SB em professores, segundo estimativas de Shirom (1989), pode estar entre $10 \%$ e $30 \%$. $\mathrm{O}$ instrumento mais utilizado para a avaliação da SB em diferentes profissões tem sido o Maslach Burnout Inventory (MBI; Maslach \& Jackson, 1986), que considera como dimensões da síndrome: baixa Realização Profissional, alta Exaustão Emocional e alta Despersonalização ou Cinismo (Maslach, Schaufeli, \& Leiter, 2001).

Utilizando o MBI para medir a prevalência de SB em profissionais da educação no Brasil, Batista et al., (2010), estudaram 265 professores da cidade de João Pessoa-PB e encontraram 33,6\% da amostra com alto nível de Exaustão Emocional, 8,3\% com alto nível de Despersonalização e 43,4\% com baixo nível de Realização Profissional. Autores de diversos países também utilizam o MBI como instrumento de avaliação de SB em professores. No Chile, Quaas (2006), avaliando a SB em professores universitários, mostrou que $16,1 \%$ têm nível reduzido, $10,2 \%$ nível moderado e 1,5\% nível elevado da SB. Na Espanha, Manassero, Vázques, Ferrer, Fornés e Fernández (2003), em um estudo com 614 professores, descobriram que quase $40 \%$ são afetados por altos níveis da SB. Em Portugal, Mota-Cardoso, Araújo, Carreira-Ramos, Gonçalves, e Ramos (2002), em estudo com 2108 professores, identificaram que $34,80 \%$ apresentavam altos níveis de Exaustão Emocional, 6,30\% de Despersonalização e $84,20 \%$ baixos níveis de Realização Profissional. Os autores concluíram que entre $6,30 \%$ e $34,80 \%$ dos professores podem estar sofrendo da SB em nível grave ou moderado. Na França, os resultados do estudo com 2558 professores mostraram que 18,1\% apresentavam Exaustão Emocional, 3,3\% Despersonalização e 31,2\% baixa Realização Profissional (Vercambre et al., 2009). Em estudo conduzido na China com 138 professores, Luk et al. (2010), encontraram 34\% da amostra com elevado nível de Exaustão Emocional, 12,3\% com elevado nível de Despersonalização, 44,2\% com baixa Realização Profissional.

Entretanto, a literatura aponta que, ainda que o instrumento MBI tenha obtido valores adequados de fidedignidade e validade (Gil-Monte, 2005b), também se detecta com frequência insuficiências psicométricas (Kristensen, Borrritz, Villadsen, \& Christensen, 2005), sobretudo quando o instrumento original é adaptado para outros idiomas, excluindo o inglês (Olmedo, Santed, Jiménez, \& Gómez, 2001; Peeters \& Rutte, 2005; Truchot, Keirsebilck, \& Meyer, 2000). Com isso, torna-se necessário o uso de instrumentos de avaliação alternativos (Kristensen et al., 2005) para a avaliação da SB, como o Cuestionario para la Evaluación del Síndrome de Quemarse por el Trabajo (CESQT; Gil-Monte, 2005a, 2011). O CESQT considera as subescalas: Ilusão pelo trabalho, Desgaste psíquico, Indolência e Culpa e tem seu modelo teórico baseado na definição de SB por Gil-Monte (2005a). Em estudos anteriores utilizando CESQT, com amostras de profissionais de enfermagem e profissionais espanhóis que trabalham com pessoas com deficiência (Gil-Monte, 2008), obtiveram-se resultados adequados de validade fatorial e de consistência interna para as suas subescalas. O questionário foi adaptado a outras culturas, obtendo resultados adequados de validade fatorial e consistência interna em pesquisas realizadas no Brasil (Gil-Monte, Carlotto, \& Câmara, 2010), Argentina (Marucco, Gil-Monte, \& Flamenco, 2007), Chile (Olivares \& Gil-Monte, 2007), México (Gil-Monte et al., 2009) e Portugal (Figueiredo-Ferraz, Gil-Monte, \& Grau-Alberola, 2012).

Estudos utilizando o CESQT como instrumento para avaliação da prevalência da SB em professores foram realizados em diferentes países. Na Catalunha-Espanha, com o modelo formativo ESTAFOR (Federació d'Ensenyament de la Unió Sindical Obrera de Catalunya, \& Universidad de Barcelona, 2007), um estudo investigou a incidência e as intervenções frente aos riscos psicossociais em 1055 professores e encontrou $16,78 \%$ da amostra com Perfil 1 e 3,70\% Perfil 2. Figueiredo-Ferraz et al. (2009) num estudo com professores portugueses encontraram $14,20 \%$ da 
amostra com altos níveis da SB (Perfil 1) e, 1,9\%, com altos níveis de SB e sentimento de Culpa (Perfil 2). No México, Unda, Sandoval e Gil-Monte (2007) encontraram SB em $17 \%$ dos professores avaliados. Dominguez et al. (2009), estudando 101 professores universitários colombianos, encontraram $10 \%$ da amostra com SB (5\% com nível leve e $5 \%$ com nível crônico) e $2 \%$ em risco de desenvolvê-la. No Brasil, a prevalência da SB, investigada em uma amostra de professores do Sul do país, é de 12\% (Perfil 1), e 5,6\% (Perfil 2; Gil-Monte et al., 2011).

No Brasil a literatura sobre SB em professores ainda é escassa, dificultando a comparação com outros estudos nacionais (Trigo, Teng, \& Hallak, 2007). Segundo Carlotto (2010) é clara a influência dos aspectos culturais e do contexto laboral sobre os resultados da SB. Portanto, é relevante a realização de pesquisas nacionais que contribuam para o diagnóstico, a intervenção e a prevenção desta patologia ocupacional. Neste sentido, este estudo é uma réplica de estudos prévios com a finalidade de avaliar e comparar a prevalência de $\mathrm{SB}$ em professores de diferentes estados brasileiros.

O presente estudo investigou a prevalência da Síndrome de Burnout em uma amostra de professores universitários brasileiros.

\section{Método}

\section{Amostra}

A amostra foi constituída por 169 professores de 7 instituições de ensino superior (2 públicas e 5 privadas) da cidade de Piracicaba-SP, que exerciam suas atividades durante o ano de 2011. Foram excluídos os que não estavam em atividade no período de coleta de dados, ou seja, inativos, em férias, afastados (licenças médica, maternidade, prêmio e por interesse particular) ou aposentados, bem como as instituições de ensino que não aceitaram participar do estudo. A taxa de resposta foi de $24,2 \%$. Com relação ao gênero, $41,4 \%$ dos participantes foram mulheres $(n=$ $70)$, e $58,6 \%$ foram homens $(n=99)$ e a média de idade foi de 45 anos $(D P=8,6 ; \operatorname{máx}=64 ; \min =28)$. Sobre o tempo de atuação profissional como professor, $18,34 \%$ da amostra exercia a docência havia menos de 4 anos, 24,26 $\%$ entre 5 e 10 anos, $14,20 \%$ entre 11 e 15 anos, $14,20 \%$ entre 16 e 20 anos e $28,99 \%$ havia mais de 20 anos. A maioria dos participantes tinha pós-graduação $(98,82 \%)$ e trabalhava em instituições públicas $(67,46 \%)$.

\section{Instrumentos}

Os níveis de SB foram avaliados pela aplicação do Questionário de Avaliação para a Síndrome de Burnout (Gil-Monte, 2005a, 2011), versão em português, validada para população brasileira (Gil-Monte et al., 2010) do Cuestionario para la Evaluación del Síndrome de Quemarse por el Trabajo (CESQT). Este instrumento, autoaplicável, é formado por 20 itens distribuídos em quatro dimensões: (a) Ilusão pelo trabalho, definida como a expectativa do indivíduo em alcançar determinadas metas laborais, pois isto supõe uma fonte de realização pessoal e profissional (cinco itens, alfa $=0,86$ ); (b) Desgaste psíquico, definido como a presença de esgotamento emocional e físico decorrente da atividade de trabalho, tendo em vista a necessidade de relacionar-se diariamente com pessoas que possuem ou geram problemas (quatro itens, alfa $=0,85$ ); (c) Indolência, definida como a presença de atitudes negativas de indiferença e cinismo frente aos clientes da organização (seis itens, alfa $=0,72$ ); (d) Culpa, definida como a ocorrência de sentimentos de culpa pelo comportamento e atitudes negativas desenvolvidas no trabalho, principalmente frente às pessoas com as quais o trabalhador deve relacionar-se profissionalmente (cinco itens, alfa $=0,81$ ). $\mathrm{O}$ valor de consistência interna alfa de Cronbach do CESQT (15 itens) foi igual a 0,85 .

A frequência das respostas, neste instrumento, é avaliada por meio de uma escala de frequência 5 pontos, variando de 0 (Nunca) a 4 (Muito frequentemente, todos os dias).

Baixas pontuações na dimensão Ilusão pelo trabalho e altas pontuações nas dimensões Desgaste psíquico e Indolência, representam altos níveis de SB. No caso dos indivíduos com o Perfil 2, as altas pontuações anteriores estão acompanhadas de altas pontuações de Culpa (Gil-Monte, 2005a, 2011).

\section{Procedimentos para Coleta dos Dados}

Os dados foram coletados de maneira não aleatória. Para a coleta dos dados, foi realizado, inicialmente, contato com a direção das instituições, sendo apresentados os objetivos do estudo a fim de obter a autorização e o apoio para a realização da pesquisa. Após esta autorização e cumprindo as normas do Comitê de Ética em Pesquisa Humana - Resolução CNS 196/96 (Conselho Nacional de Saúde, 1997), o instrumento foi entregue aos professores pela secretária de cada departamento das instituições participantes. Os documentos eram enviados em 2 envelopes, sendo que um continha a carta convite para a pesquisa e o questionário de investigação, e o outro, o Termo de Consentimento Livre e Esclarecido. O recolhimento dos envelopes foi realizado pela pesquisadora, junto à secretaria de departamento de cada instituição, 10 dias após a entrega.

\section{Procedimentos para Análise dos Dados}

Os dados foram registrados e analisados no Programa de Estatística para Ciências Sociais (SPSS - Statistical Package of Social Science, versão 20).

Para se analisar o nível da SB na amostra, foram utilizados os 5 níveis propostos por Gil-Monte (2011), de acordo com os percentis 10, 33, 66 e 90 (P10, P33, P66 e P90). Assim sendo, foram classificados como: (a) muito baixo - pontuações menores ou iguais ao P10; (b) baixo - pontuações menores ou iguais ao P33; (c) médio - pontuações menores ou iguais ao P66; (d) alto - pontuações menores que P90 e (e) crítico - pontuações maiores ou iguais ao $\mathrm{P} 90$.

Para identificar os casos de Perfil 1 e Perfil 2, foram utilizados os critérios do manual do CESQT (Gil-Monte, 
Costa, L. S. T., Gil-Monte, P. R., Possobon, R. F.\& Ambrosano, G. M. B. (2013). Prevalência da Síndrome de Burnout em uma Amostra de Professores Universitários Brasileiros.

2011). Foram considerados Perfil 1 os casos que apresentaram pontuações iguais ou superiores ao P90 na pontuação média dos 15 itens que formam as subescalas de Ilusão pelo trabalho (invertida), Desgaste psíquico e Indolência, mas inferiores ao P90 na subescala de Culpa. No Perfil 2 foram incluídos os casos com pontuações iguais ou superiores ao P90 na pontuação média dos 15 itens citados acima e também iguais ou superiores ao P90 na subescala de Culpa.

\section{Resultados e Discussão}

A Tabela 1 mostra as médias, desvio padrão, variação e consistência interna das dimensões do questionário utilizado neste estudo.

Tabela 1

Média, Desvio Padrão (DP), Variação e Consistência Interna (alfa de Cronbach) das Dimensões do CESQT

\begin{tabular}{lccc}
\hline & Média $(D P)$ & Variação & alfa de Cronbach \\
\hline Ilusão pelo trabalho & $2,93(0,81)$ & $0-4$ & 0,86 \\
Desgaste psíquico & $1,67(1,00)$ & $0-4$ & 0,85 \\
Indolência & $0,68(0,56)$ & $0-4$ & 0,72 \\
Culpa & $0,82(0,64)$ & $0-4$ & 0,81 \\
CESQT (15 itens) & $1,10(0,59)$ & $0-4$ & 0,85 \\
\hline
\end{tabular}

Todas as dimensões do questionário apresentaram valores de consistência interna alfa de Cronbach superiores a 0,70 , valores adequados segundo Carretero-Dios e Pérez (2007): Ilusão pelo trabalho, alfa $=0,86$; Desgaste psíquico, alfa $=0,85$; Indolência, alfa $=0,72 ;$ e Culpa, alfa $=0,81$ (Tabela 1). Portanto, pode-se a firmar que todos os itens estão relacionados significativamente com seus construtos originais para avaliar a mesma faceta da SB. Estes resultados também foram obtidos por Gil-Monte et al. (2010) no estudo de avaliação da validade fatorial e de construto da versão brasileira do CESQT e, por Gil-Monte et al. (2011) com uma amostra de docentes da região Sul do Brasil.

Sobre as médias das dimensões, o valor mais alto foi obtido para a Ilusão pelo trabalho $(M=2,93 ; D P=0,81)$, cujos itens, diferentemente dos demais do CESQT, estão formulados em sentido positivo (altas pontuações são indicativas de baixos níveis de $\mathrm{SB}$ ). $\mathrm{O}$ valor mais baixo foi obtido para a dimensão de Indolência $(M=0,68 ; D P$ $=0,56$ ).

Seguindo os critérios de classificação da SB de GilMonte (2011) foram aplicados os P10, P33, P66 e P90 do manual do CESQT na amostra estudada para cada dimensão. Os resultados apontaram que 11,2\% $(n=19)$ dos professores universitários avaliados apresentaram Perfil 1 da SB, e 3\% (n=5) Perfil 2. Mais especificamente, são considerados casos críticos em cada dimensão aqueles professores que apresentaram nível muito baixo de Ilusão pelo trabalho $(16,6 \% ; n=28)$, níveis críticos de Desgaste psíquico $(17,8 \%, n=30)$, de Indolência $(5,9 \%, n=10)$, e de Culpa $(8,3 \%, n=14$; Tabela 2$)$.

Tabela 2

Frequência e Porcentagem de Professores Universitários com Niveis Muito Baixo, Baixo, Médio, Alto e Crítico da Sindrome de Burnout (SB) de acordo com os Percentis (P) do Manual do CESQT

\begin{tabular}{lrrrrr}
\hline & Muito baixo & Baixo & Médio & Alto & Crítico \\
\hline Ilusão pelo trabalho & $P \leq 10$ & $P 11-\mathrm{P} 33$ & $P 34-\mathrm{P} 66$ & $P 67-\mathrm{P} 89$ & $P \geq 90$ \\
Desgaste psíquico & $28(16,6 \%)$ & $61(36,1 \%)$ & $47(27,8 \%)$ & $14(8,3 \%)$ & $19(11,2 \%)$ \\
Indolência & $14(8,3 \%)$ & $46(27,2 \%)$ & $59(34,9 \%)$ & $20(11,8 \%)$ & $30(17,8 \%)$ \\
Culpa & $26(15,4 \%)$ & $45(26,6 \%)$ & $63(37,3 \%)$ & $25(14,8 \%)$ & $10(5,9 \%)$ \\
CESQT (15 itens) & $24(14,2 \%)$ & $53(31,4 \%)$ & $45(26,6 \%)$ & $33(19,5 \%)$ & $14(8,3 \%)$ \\
& $19(11,2 \%)$ & $36(21,3 \%)$ & $53(31,4 \%)$ & $42(24,9 \%)$ & $19(11,2 \%)$
\end{tabular}

A prevalência de SB encontrada nesta amostra de professores universitários da cidade de Piracicaba (região Sudeste do Brasil; Perfil $1=11,2 \%$ e Perfil $2=3 \%$ ) é muito similar aquela encontrada no estudo de Gil-Monte et al. (2011) com professores da cidade de Porto Alegre (região Sul do Brasil). Nesta amostra de 714 profissionais, $12 \%$ dos participantes apresentavam altos níveis de SB (Perfil 1), e 5,6\% tinham a forma mais grave da SB (Perfil 2). Baseados em considerações psicométricas, os casos identificados como Perfil 2, ou seja, profissionais 
que apresentam baixa Ilusão pelo trabalho, altos níveis de Desgaste psíquico e Indolência acompanhados de sentimentos de Culpa, podem ser considerados como casos de SB pela legislação brasileira.

Os resultados encontrados nesta amostra de professores do sudeste brasileiro também são semelhantes aos de Figueiredo-Ferraz et al. (2009) que, em uma amostra de 211 professores de diferentes instituições portuguesas, verificaram a prevalência de $14,2 \%$ de Perfil 1 e de $1,9 \%$ de Perfil 2. Há também coincidência com os resultados de Gil-Monte, Carretero, Roldán e Núñez-Román (2005) que, no estudo com 154 profissionais que trabalham com pessoas com deficiência, encontraram $11,7 \%$ de Perfil 1 e 1,30\% de Perfil 2. Por outro lado, a prevalência de SB destes professores brasileiros é diferente daquela obtida no estudo de Unda et al. (2007) com uma amostra de 698 professores mexicanos (Perfil $=35,50 \%$ e Perfil 2 $=17,20 \%$ ). Estes resultados podem estar relacionados com a diferença nos aspectos cultural e organizacional (Golembiewski, Scherb, \& Boudreau, 1993; Maslach et al., 2001) ou podem ser atribuídas a diferenças na seleção das amostras.

Considerando as dimensões do CESQT, merece atenção àquela porcentagem de professores que apresentou nível baixo de Ilusão pelo trabalho $(36,1 \%, n=61)$ e níveis altos de Desgaste psíquico $(11,8 \%, n=20)$, Indolência $(14,8 \%, n=25)$ e Culpa $(19,5 \%, n=33)$ (Tabela 2). Estas prevalências podem indicar uma tendência para o aparecimento dos casos de SB depois de um período de exposição a estressores.

Para Carlotto e Câmara (2007), os docentes universitários investem em sua carreira acadêmica e, quando obtêm satisfação com este processo de crescimento, apresentam maiores índices de realização no trabalho. Nesta amostra de professores universitários pode-se observar que uma porcentagem importante apresenta nível muito baixo $(16,6 \%$; $n=28)$ e baixo $(36,1 \% ; n=61)$ de Ilusão pelo trabalho, o que pressupõe uma baixa realização pessoal e profissional destes professores.

A SB tem implicações para a saúde física, mental e social do indivíduo afetado (Batista et al., 2010). De acordo com dados do Ministério da Saúde (2008), a taxa de incidência de doenças relacionadas ao trabalho vem aumentando nos últimos anos $(2003=8,8 \% ; 2004=$ 10,5\%; $2005=12,3 \%$ ), e o Estado de São Paulo apresenta a $4^{\mathrm{a}}$ maior taxa nacional $(2003=13,25 \% ; 2004=15,86 \%$; $2005=15,93 \%$ ). Na realidade, estas porcentagens podem ser ainda maiores por haver subnotificação das doenças ocupacionais no Brasil (Rêgo, 1994; Salim, 2003) e pela dificuldade no diagnóstico de doenças mentais relacionadas ao trabalho. Além disso, a SB tem repercussões no sistema educacional, na aprendizagem, na motivação e também no comportamento dos alunos (Rudow, 1999).

Destaca-se, como limitação deste estudo, a possibilidade de ter ocorrido um viés de seleção, uma vez que, os professores participantes foram voluntários e que, portanto, existe a possibilidade de aqueles docentes mais afetados não terem participado.

Tendo em vista estes resultados, seria importante a realização de novas pesquisas com amostras maiores e de diferentes regiões do país, utilizando o instrumento CESQT, com o objetivo de replicar estes resultados e mapear a situação da SB em professores do Brasil.

\section{Considerações Finais}

A incidência da SB em diversos países teve um aumento importante nos últimos anos e a literatura estima que de $4 \%$ a $7 \%$ dos trabalhadores são afetados por SB (Melamed et al., 2006). Neste estudo com professores brasileiros a prevalência da SB é semelhante à encontrada na literatura o que nos permite afirmar que o problema tem uma dimensão similar.

Uma das contribuições práticas deste estudo é documentar a prevalência de SB no Brasil, replicando estudos prévios, e sua similaridade com as taxas de prevalência internacionais. Baseados nestas prevalências, Melamed et al., (2006) consideram que a SB é um importante problema de saúde pública, afetando a saúde mental e física dos indivíduos, sendo portanto, um motivo de preocupação para os formadores de políticas de saúde. Nossos resultados deveriam ser considerados para fomentar políticas e ações de prevenção de estresse laboral e SB em docentes. Concordamos com as recomendações de outros autores e acreditamos que investir na saúde e na qualidade de vida do professor é também investir numa educação de qualidade.

\section{Referências}

Antoniou, A. S., Polychroni, F., \& Vlachakis, A. N. (2006). Gender and age differences in occupational stress and professional burnout between primary and high-school teachers in Greece. Journal of Managerial Psychology, 21, 682-690.

Batista, J. B. V., Carlotto, M. S., Coutinho, A. S., \& Augusto, L. G. S. (2010). Prevalência da Síndrome de Burnout e fatores sociodemográficos e laborais em professores de escolas municipais da cidade de João Pessoa, PB. Revista Brasileira de Epidemiologia, 13, 502-512.

Betoret, F. D. (2006). Stressors, self-efficacy, coping resources, and burnout among secondary school teachers in Spain. Educational Psychology, 26, 519-539.

Burke, R. J., Greenglass, E. R., \& Schwarzer, R. (1996). Predicting teacher burnout over time: Effects of work stress, social support, and self-doubts on burnout and its consequences. Anxiety, Stress and Coping: An International Journal, 9, 261-275.

Carlotto, M. S. (2002). A Síndrome de Burnout e o trabalho docente. Psicologia em Estudo, 7, 21-29.

Carlotto, M. S. (2010). Síndrome de Burnout diferenças segundo níveis de ensino. Psico, 41, 495-502.

Carlotto, M. S., \& Câmara, S. G. (2007). Propriedades psicométricas do Maslach Burnout Inventory em uma amostra multifuncional. Estudos de Psicologia (Campinas), 24, 325-332. 
Costa, L. S. T., Gil-Monte, P. R., Possobon, R. F.\& Ambrosano, G. M. B. (2013). Prevalência da Síndrome de Burnout em uma Amostra de Professores Universitários Brasileiros.

Carretero-Dios, H., \& Pérez, C. (2007). Standards for the development and review of instrumental studies: Considerations about test selection in psychological research. International Journal of Clinical and Health Psychology, 7, 863-882.

Conselho Nacional de Saúde. (1997). Diretrizes e normas para pesquisa envolvendo seres humanos. Resolução CNS 196/196. Brasília, DF: Autor.

Decreto $n^{\circ}$ 3.048, de 6 de maio de 1999. (1999, 7 maio). Aprova o Regulamento da Previdência Social, e dá outras providências. Diário Oficial da União.

Dominguez, C. C., Guitiérrez, O. G., Anguila, D. M., Barrios, C. L., Barrero, Y. B., \& Medrano, C. V. (2009). Prevalencia del Síndrome del Burnout y su correlación com factores psicosociales em docentes de uma institución universitária privada de la ciudad de Barranquilla. Psicogente, 12, 142-157.

Federació d'Ensenyament de la Unió Sindical Obrera de Catalunya, \& Universidad de Barcelona (2007). Estafor. Estrategias de afrontamiento del éstres para docentes. Modelo formativo de intervención delante los riesgos psicosociales en el ámbito educativo. Recuperado em http://www.conforcat. cat/AACC/PRODUCTES\%20FINALS/AC20060028\%20 DEFINICI\%C3\%93\%20DEL\%20PERFIL\%20DEL\%20 MEDIADOR\%20I\%20DISSENY\%20DE\%20METODOLOGIES\%20AVALUACI\%C3\%93\%20I\%20DESENVOLUPAMENT\%20DE\%20COMPET\%C3\%88NCIES/ DOCUMENTS/2-4-5\%20-\%20S\%C3\%8DNTESIS $\% 20$ ESTAFOR\%20(castellano).pdf

Figueiredo-Ferraz, H., Gil-Monte, P. R., \& Grau-Alberola, E. (2009). Prevalence of burnout syndrome in Portuguese teachers. Aletheia, 29, 6-15.

Figueiredo-Ferraz, H., Gil-Monte, P. R., \& Grau-Alberola, E. (2013). Psychometric properties of the "Spanish Burnout Inventory" (SBI): Adaptation and validation in a Portuguesespeaking sample. European Review of Applied Psychology, 63, 33-40.

Gil-Monte, P. R. (2005a). El sindrome de quemarse por el trabajo ("Burnout"). Una enfermedad laboral en la sociedad del bienestar. Madrid, España: Pirámide.

Gil-Monte, P. R. (2005b). Factorial validity of the Maslach Burnout Inventory (MBI-HSS) among Spanish professionals. Revista de Saúde Pública, 39, 1-8.

Gil-Monte, P. R. (2008). Magnitude of relationship between burnout and absenteeism: A preliminary study. Psychological Reports, 102, 465-468.

Gil-Monte, P. R. (2011). CESQT. Cuestionario para la evaluación del Síndrome de Quemarse por el Trabajo. Manual. Madrid, España: TEA.

Gil-Monte, P. R., Carlotto, M. S., \& Câmara, S. (2010). Validation of the Brazilian version of the "Spanish Burnout Inventory" in teachers. Revista de Saúde Pública, 44, 140-147.

Gil-Monte, P. R., Carlotto, M. S., \& Câmara, S. (2011). Prevalence of burnout in a sample of Brazilian teachers. The European Journal of Psychiatry, 25, 205-212.

Gil-Monte, P. R., Carretero, N., Roldán, M. D., \& NúñezRomán, E. (2005). Prevalencia del síndrome de quemarse por el trabajo (Burnout) en monitores de taller para personas con discapacidad. Revista de Psicología del Trabajo y de las Organizaciones, 21, 107-123.

Gil-Monte, P. R., Unda, S., \& Sandoval, J. I. (2009). Validez factorial del "Cuestionario para la Evaluación del Síndrome de Quemarse por el Trabajo" (CESQT) en una muestra de maestros mexicanos. Salud Mental, 32, 205-214.
Golembiewski, R. T., Scherb, K., \& Boudreau, A. (1993). Burnout in cross-national settings: Generic and model-specific perspectives. In W. B. Schaufeli, C. Maslach, \& T. Marek (Eds.), Professional Burnout: Recent developments in theory and research (pp. 217-236). London: Taylor \& Francis.

Hakanen, J. J., Bakker, A. B., \& Schaufeli, W. B. (2005). Burnout and work engagement among teachers. Journal of School Psychology, 43, 495-513.

Kokkinos, C. M. (2007). Job stressors, personality and burnout in primary school teachers. British Journal of Educational Psychology, 77, 229-243.

Kristensen, T. S., Borrritz, M., Villadsen, E., \& Christensen, K. B. (2005). The Copenhagen Burnout Inventory: A new tool for the assessment of burnout. Work Stress, 19, 192-207.

Luk, A. L., Chan, B. P. S., Cheong, S. W., \& Ko, S. K. K. (2010). An exploration of the burnout situation on teachers in two schools in Macau. Social Indicators Research, 95, 489-502.

Manassero, M., Vázquez, A., Ferrer, V., Fornés, J., \& Fernández, M. (2003). Estrés y Burnout en la enseñanza. Palma de Mallorca, España: Ediciones Universitat de les Illes Balears.

Marqués-Pinto, A., Lima, M. L., \& Lopes da Silva, A. (2005). Fuentes de estrés, Burnout y estrategias de coping en profesores portugueses. Revista de Psicología del Trabajo y de las Organizaciones, 21, 125-143.

Marucco, M. A., Gil-Monte, P. R., \& Flamenco, E. (2007). Síndrome de quemarse por el trabajo (Burnout) en pediatras de hospitales generales, estudio comparativo de la prevalencia medida con el MBI-HSS y el CESQT. Información Psicologica, 91-92, 32-42.

Maslach, C., \& Jackson, S. E. (1986). Maslach Burnout Inventory $\left(2^{\text {nd }}\right.$ ed). Palo Alto, CA: Consulting Psychologists Press.

Maslach, C., Schaufeli, W. B., \& Leiter, M. P. (2001). Job burnout. Annual Review Psychology, 52, 397-422.

Melamed, S., Shirom, A., Toker, S., Berliner, S., \& Shapira, I. (2006). Burnout and risk of cardiovascular disease: Evidence, possible causal paths, and promising research directions. Psychological Bulletin, 132, 327-353.

Ministério da Saúde. (2001). Doenças relacionadas ao trabalho. Manual de procedimentos para os serviços de saúde. Brasília, DF: Autor. Recuperado em http://dtr2001.saude.gov.br/ editora/produtos/livros/pdf/02_0388_M1.pdf

Ministério da Saúde. (2008). REDE Interagencial de Informação para a Saúde. Indicadores básicos para a saúde no Brasil. Conceitos e aplicações (2. ed.). Brasília, DF: Organização Pan-Americana da Saúde.

Mota-Cardoso, R., Araújo, A., Carreira-Ramos, R., Gonçalves, G., \& Ramos, M. (2002). O stress nos professores portugueses - Estudo IPSSO 2000. Porto, Portugal: Porto Editora.

Organização Internacional do Trabalho. (1981). Emploi et conditions de travail des enseignants. Geneve, Switzerland: Bureau International du Travail.

Olivares, V. E., \& Gil-Monte, P. R. (2007). Análisis de las propiedades psicométricas del "Cuestionario para la Evaluación del Síndrome de Quemarse por el Trabajo” (CESQT) en profesionales chilenos. Ansiedad y Estrés, 13, 229-240.

Olmedo, M., Santed, M. A., Jiménez, R., \& Gómez, M. D. (2001). El Síndrome de Burnout: variables laborales, personales y psicopatológicas asociadas. Psiquis, 22, 117-129.

Peeters, M. A., \& Rutte, C. G. (2005). Time management behavior as a moderator for the job demand-control interaction. Journal of Occupational Health Psychology, 10, 64-75. 
Quaas, C. (2006). Diagnóstico de Burnout y técnicas de afrontamiento al estrés en profesores universitarios de la Quinta Región de Chile. Revista Psico-Perspectivas, 1, 65-75.

Rêgo, M. A. (1994). Acidentes e doenças do trabalho no Estado da Bahia, de 1970 a 1992. Revista Brasileira de Saúde Ocupacional, 22, 21-31.

Rudow, B. (1999). Stress and burnout in the teaching profession: European studies, issues, and research perspectives. In R. Vanderbergue \& M. A. Huberman (Eds.), Understanding and preventing teacher burnout: A source book of international practice and research (pp. 38-58). Cambridge, UK: Cambridge University Press.

Salim, C. A. (2003). Doenças do trabalho: Exclusão, segregação e relações de gênero. São Paulo Perspectiva, 17, 11-24.

Shirom, A. (1989). Burnout in work organizations. In C. L. Cooper \& I. Robertson (Eds.), International Review of Industrial and Organizational Psychology (pp. 25-48). New York: Wiley \& Sons.

Skaalvik, E. M., \& Skaalvik, S. (2007). Dimensions of teacher self-efficacy and relations with strain factors, perceived collective teacher efficacy, and teacher burnout. Journal of Educational Psychology, 99, 611-625.

Trigo, T. R., Teng, C. T., \& Hallak, J. E. (2007). Síndrome de Burnout ou estafa profissional e os transtornos psiquiátricos. Revista de Psiquiatria Clínica, 34, 223-233.

Truchot, D., Keirsebilck, L., \& Meyer, S. (2000). Communal orientation may not buffer burnout. Psychological Reports, 86, 872-878.

Unda, S., Sandoval, J. I., \& Gil-Monte, P. R. (2007). Prevalencia del síndrome de quemarse por el trabajo (SQT; Burnout) em maestros mexicanos. Informació Psicologica, 91-92, 53-63.

Vercambre, M., Brosselin, P., Gilbert, F., Nerrière, E., \& KovessMasféty, V. (2009). Individual and contextual covariates of burnout: A cross-sectional nationwide study of French teachers. BioMed Central Public Health, 9, 333. 